\title{
Correction to: In-Silico analyses of Nonsynonymous Variants in the BRCA1 Gene
}

\author{
Sidra Arshad ${ }^{1} \cdot$ Irfan Ishaque $^{1} \cdot$ Sidra Mumtaz $^{1} \cdot$ Muhammad Usman Rashid $^{2}$. \\ Naila Malkani ${ }^{1}$ D
}

Published online: 27 November 2021

(c) Springer Science+Business Media, LLC, part of Springer Nature 2021

\section{Correction to: Biochemical Genetics (2021) 59:1506-1526 https://doi.org/10.1007/s10528-021-10074-7}

In this article Table 1 and 2 did not use the mentioned (in materials and methods) nucleotide sequence of the human BRCA1 gene (NM_007294.3) (ID P38398) for the analysis that's why some of the amino acids do not correspond to the residue in P38398 and NM_007294.3. But this discrepancy is removed in the below given Table 1 and 2 and can be replaced with the Table 1 and 2 of the article.

The original article can be found online at https://doi.org/10.1007/s10528-021-10074-7.

Naila Malkani

nailamalkani@gcu.edu.pk

1 Department of Zoology, GC University, Lahore, Pakistan

2 Department of Basic Sciences Research, Shaukat Khanum Memorial Cancer Hospital and Research Centre (SKMCH\&RC), Lahore, Pakistan 
Table 1 SIFT analyses prediction of 122 BRCA1 nsSNPs

\begin{tabular}{|c|c|c|c|c|}
\hline & rs Ids & Variant type & Codon change & Amino acid \\
\hline 1 & rs41293455 & $\mathrm{M}$ & CTG $[\mathrm{C} / \mathrm{G}] \mathrm{GA}$ AAT & R1443G \\
\hline 2 & rs80357164 & M & AAG [T/A]GT GAC & $\mathrm{C} 39 \mathrm{~S}$ \\
\hline 3 & rs80357025 & M & GGG C $[\mathrm{C} / \mathrm{G}] \mathrm{C}$ TTC & P1771R \\
\hline 4 & rs28897672 & M & CAG $[\mathrm{T} / \mathrm{G}] \mathrm{GT} \mathrm{CCT}$ & C61G \\
\hline 5 & rs28897672 & M & CAG $[\mathrm{T} / \mathrm{C}] \mathrm{GT} \mathrm{CCT}$ & C61R \\
\hline 6 & rs55770810 & M & GAA $[\mathrm{C} / \mathrm{T}] \mathrm{GG}$ ACA & R1699W \\
\hline 7 & rs41293461 & M & TTC T[G/C $] \mathrm{G}$ GTG & W1718S \\
\hline 8 & rs41293463 & M & $\mathrm{AAC} A[\mathrm{~T} / \mathrm{G}] \mathrm{G} \mathrm{CCC}$ & M1775R \\
\hline 9 & rs41293463 & M & $\mathrm{AAC} A[\mathrm{~T} / \mathrm{A}] \mathrm{G} \mathrm{CCC}$ & M1775K \\
\hline 10 & rs45553935 & M & GAA G[T/G]C AGA & V1736G \\
\hline 11 & rs45553935 & M & GAA G[T/C]C AGA & V1736A \\
\hline 12 & rs55650082 & M & CTC [G/A]AA TTA & E597K \\
\hline 13 & rs55851803 & M & TTA T[G/A]T AAG & C64Y \\
\hline 14 & rs80356860 & M & CTA G[G/C]A ATT & G1706A \\
\hline 15 & rs80356862 & M & ACT G[C/G]T GGG & A1623G \\
\hline 16 & rs 80356880 & M & TCC A[C/G]A AAG & $\mathrm{T} 37 \mathrm{R}$ \\
\hline 17 & rs80356890 & M & ACT $[\mathrm{A} / \mathrm{G}] \mathrm{CT}$ CAT & $\mathrm{T} 1685 \mathrm{~A}$ \\
\hline 18 & rs80356907 & M & GAA [G/C]AG ATA & E649Q \\
\hline 19 & rs80356914 & M & GAG TG[G/T] GTG & W1837C \\
\hline 20 & rs80356915 & M & AGT [G/C $]$ AA AGA & E445Q \\
\hline 21 & rs80356942 & M & CGA [G/A]AG TGG & E1836K \\
\hline 22 & rs80356945 & M & ACT $[\mathrm{C} / \mathrm{A}] \mathrm{AG}$ GAA & Q780K \\
\hline 23 & rs80356950 & M & CTT [G/A]AA CTA & E624K \\
\hline 24 & rs80356988 & M & CTA [G/C]AG GGA & E1559Q \\
\hline 25 & rs80356991 & M & CCC [G/A]AA AAT & E143K \\
\hline 26 & rs80357003 & M & GTT T[C/T $]$ A AAG & S864L \\
\hline 27 & rs80357005 & M & ACT [G/A]AA TTG & E638K \\
\hline 28 & rs 1064796143 & M & GGA A[T/C]T GCG & I1707T \\
\hline 29 & rs80357061 & M & GTT A[T/G]G AAA & M1689R \\
\hline 30 & rs80357061 & M & GTT A[T/C $]$ G AAA & M1689T \\
\hline 31 & rs80357064 & M & TTA [T/G]GT AAG & C64G \\
\hline 32 & rs80357065 & $\mathrm{M}$ & CTG [T/A]GT GGT & C1787S \\
\hline 33 & rs80357069 & M & TGT G[G/A]T GCT & G1788D \\
\hline 34 & rs80357093 & M & CAG T[G/A]T CCT & C61Y \\
\hline 35 & rs80357104 & M & CAG T[C/T]T ATT & S1722F \\
\hline 36 & rs80357107 & M & TGG G[T/A]G TTG & V1838E \\
\hline 37 & rs80357111 & M & $\mathrm{A}[\mathrm{T} / \mathrm{C}] \mathrm{G}$ GAT & M1T \\
\hline 38 & rs80357147 & M & CTT [A/G]AA GAA & K719E \\
\hline 39 & rs 80357150 & M & TTT T[G/T $] \mathrm{C}$ ATG & $\mathrm{C} 47 \mathrm{~F}$ \\
\hline 40 & rs80357167 & M & ACT [G/A]AG CCA & E489K \\
\hline 41 & rs 80357202 & M & TTG [G/A]AA GAA & E1352K \\
\hline 42 & rs142074233 & M & AAT [C/G]AG GGA & Q534E \\
\hline
\end{tabular}


Table 1 (continued)

\begin{tabular}{|c|c|c|c|c|}
\hline & rs Ids & Variant type & Codon change & Amino acid \\
\hline 43 & rs273897656 & M & GCT T[T/C $]$ A ATA & L440S \\
\hline 44 & rs397507206 & M & AAG T[C/T $]$ A TTT & S988L \\
\hline 45 & rs397507239 & M & ATG $[\mathrm{A} / \mathrm{C}] \mathrm{AA}$ ACA & K1690Q \\
\hline 46 & rs80357212 & M & CAT [G/A]CA ATT & A1823T \\
\hline 47 & rs80357222 & M & GTT $[\mathrm{A} / \mathrm{T}] \mathrm{GC}$ TAT & $\mathrm{S} 1715 \mathrm{C}$ \\
\hline 48 & rs80357239 & M & TTC TG[G/T] GTG & W1718C \\
\hline 49 & rs80357251 & M & GCA [G/A]AA GAG & E879K \\
\hline 50 & rs80357253 & M & GCC $[\mathrm{A} / \mathrm{G}] \mathrm{AA}$ GTA & K408E \\
\hline 51 & rs80357276 & M & GAC C $[\mathrm{A} / \mathrm{G}] \mathrm{C}$ ATA & $\mathrm{H} 41 \mathrm{R}$ \\
\hline 52 & rs80357281 & M & GGG C[T/C $]$ A GAA & L1764P \\
\hline 53 & rs80357269 & M & TTA T[C/T]A TTG & S1262L \\
\hline 54 & rs80357287 & M & [A/G]TG GAT & M1V \\
\hline 55 & rs80357309 & M & TTA [G/C]AA CAG & E1419Q \\
\hline 56 & rs80357327 & M & TTT [T/A]GC AAA & $\mathrm{C} 44 \mathrm{~S}$ \\
\hline 57 & rs80357370 & M & TTT [T/G]GC ATG & $\mathrm{C} 47 \mathrm{G}$ \\
\hline 58 & rs80357382 & M & AAA $[\mathrm{A} / \mathrm{G}] \mathrm{GG}$ AGC & $\mathrm{R} 71 \mathrm{G}$ \\
\hline 59 & rs80357389 & M & GAA A[G/T]G TCA & $\mathrm{R} 1495 \mathrm{M}$ \\
\hline 60 & rs80357389 & M & GAA A[G/A]G TCA & R1495K \\
\hline 62 & rs80357438 & M & ATC T[T/C $]$ A GAG & L22S \\
\hline 63 & rs80357446 & M & TTT T[G/T]C AAA & $\mathrm{C} 44 \mathrm{~F}$ \\
\hline 64 & rs80357446 & M & TTT T[G/A]C AAA & $\mathrm{C} 44 \mathrm{Y}$ \\
\hline 65 & rs80357450 & M & AGA G[G/A]A GAT & G1738E \\
\hline 66 & rs80357463 & M & GAA A[T/G]C TGT & $\mathrm{I} 1766 \mathrm{~S}$ \\
\hline 67 & rs80357475 & M & AT[G/T] GAT & M1I \\
\hline 68 & rs397508902 & M & CTC [G/A]AA AAA & E575K \\
\hline 69 & rs80357498 & M & AAG T[G/A]T GAC & C39Y \\
\hline 70 & rs80356898 & $\mathrm{N}$ & ATT $[\mathrm{C} / \mathrm{T}] \mathrm{AG}$ AAT & $\mathrm{Q}_{563}^{\mathrm{a}}$ \\
\hline 71 & rs28897686 & $\mathrm{N}$ & ACC $[\mathrm{G} / \mathrm{T}] \mathrm{AG}$ TGT & $\mathrm{E} 1250^{\mathrm{a}}$ \\
\hline 72 & rs41286296 & $\mathrm{N}$ & GGA [G/T]AA AGG & $\mathrm{E} 755^{\mathrm{a}}$ \\
\hline 73 & rs41293455 & $\mathrm{N}$ & CTG $[\mathrm{C} / \mathrm{T}] \mathrm{GA}$ AAT & $\mathrm{R} 1443^{\mathrm{a}}$ \\
\hline 74 & rs41293461 & $\mathrm{N}$ & TTC T[G/A]G GTG & $\mathrm{W} 1718^{\mathrm{a}}$ \\
\hline 75 & rs55650082 & $\mathrm{N}$ & CTC [G/T]AA TTA & $\mathrm{E} 597^{\mathrm{a}}$ \\
\hline 76 & rs80357429 & $\mathrm{N}$ & TCT T[C/T $]$ A ACC & S1596L \\
\hline 76 & rs56329598 & $\mathrm{N}$ & GTT [A/T]AA GTG & $\mathrm{K} 739^{\mathrm{a}}$ \\
\hline 77 & rs62625306 & $\mathrm{N}$ & ACA [G/T]AA CCA & E797 ${ }^{\mathrm{a}}$ \\
\hline 78 & rs80356864 & $\mathrm{N}$ & $\mathrm{AAC}[\mathrm{C} / \mathrm{T}] \mathrm{AG}$ AAG & $\mathrm{Q} 54^{\mathrm{a}}$ \\
\hline 79 & rs80356866 & $\mathrm{N}$ & TCT $[\mathrm{C} / \mathrm{T}] \mathrm{AG}$ GAA & $\mathrm{Q} 1281^{\mathrm{a}}$ \\
\hline 80 & rs80356868 & $\mathrm{N}$ & ACA [G/T]AG GAC & $\mathrm{E} 1817^{\mathrm{a}}$ \\
\hline 81 & rs80356873 & $\mathrm{N}$ & TAC $[\mathrm{C} / \mathrm{T}] \mathrm{AG}$ TGC & $\mathrm{Q} 1846^{\mathrm{a}}$ \\
\hline 82 & rs80356875 & $\mathrm{N}$ & AAA $[\mathrm{G} / \mathrm{T}] \mathrm{AA}$ TTT & $\mathrm{E} 720^{\mathrm{a}}$ \\
\hline 83 & rs80356914 & $\mathrm{N}$ & GAG TG[G/A] GTG & $\mathrm{W} 1837^{\mathrm{a}}$ \\
\hline 84 & rs80356915 & $\mathrm{N}$ & AGT [G/T]AA AGA & $\mathrm{E} 445^{\mathrm{a}}$ \\
\hline
\end{tabular}


Table 1 (continued)

\begin{tabular}{|c|c|c|c|c|}
\hline & rs Ids & Variant type & Codon change & Amino acid \\
\hline 85 & rs80356923 & $\mathrm{N}$ & GAA $[\mathrm{G} / \mathrm{T}] \mathrm{AG}$ AAC & E1214 ${ }^{\mathrm{a}}$ \\
\hline 86 & rs 80356928 & $\mathrm{~N}$ & ACG $[\mathrm{A} / \mathrm{T}] \mathrm{AA}$ GCT & $\mathrm{K} 583^{\mathrm{a}}$ \\
\hline 87 & rs 80356935 & $\mathrm{~N}$ & GAA TG[G/A] AAT & W353 ${ }^{\mathrm{a}}$ \\
\hline 88 & rs80356942 & $\mathrm{N}$ & CGA $[\mathrm{G} / \mathrm{T}] \mathrm{AG}$ TGG & $\mathrm{E} 1836^{\mathrm{a}}$ \\
\hline 89 & rs 80356945 & $\mathrm{~N}$ & ACT $[\mathrm{C} / \mathrm{T}] \mathrm{AG}$ GAA & Q780 \\
\hline 90 & rs 80356950 & $\mathrm{~N}$ & CTT [G/T]AA CTA & E624 ${ }^{\mathrm{a}}$ \\
\hline 91 & rs 80356982 & $\mathrm{~N}$ & AGT $[\mathrm{C} / \mathrm{T}] \mathrm{AG}$ TGT & Q804 ${ }^{\mathrm{a}}$ \\
\hline 92 & rs80356991 & $\mathrm{N}$ & $\mathrm{CCC}[\mathrm{G} / \mathrm{T}] \mathrm{AA}$ AAT & $\mathrm{E} 143^{\mathrm{a}}$ \\
\hline 93 & rs 80357011 & $\mathrm{~N}$ & $\mathrm{CAG}[\mathrm{C} / \mathrm{T}] \mathrm{AG}$ AGG & Q1396 ${ }^{\mathrm{a}}$ \\
\hline 94 & rs 80357035 & $\mathrm{~N}$ & TGT [G/T]AA CAA & E904* \\
\hline 95 & rs 80357067 & $\mathrm{~N}$ & GAA $[\mathrm{C} / \mathrm{T}] \mathrm{AA}$ AGC & $\mathrm{Q} 1447^{\mathrm{a}}$ \\
\hline 96 & rs80357068 & $\mathrm{N}$ & GAC T[C/G]A CAT & $\mathrm{S} 398^{\mathrm{a}}$ \\
\hline 97 & rs 80357083 & $\mathrm{~N}$ & AAT [G/T]AG GTA & $\mathrm{E} 418^{\mathrm{a}}$ \\
\hline 98 & rs80357136 & $\mathrm{N}$ & GAA $[\mathrm{C} / \mathrm{T}] \mathrm{AG} \mathrm{CCT}$ & Q1135 ${ }^{\mathrm{a}}$ \\
\hline 99 & rs 80357148 & $\mathrm{~N}$ & GTG [G/T]AA AGG & E1494 ${ }^{\mathrm{a}}$ \\
\hline 100 & rs 80357204 & $\mathrm{~N}$ & TAC $[\mathrm{A} / \mathrm{T}] \mathrm{AG}$ TTT & $\mathrm{K} 1667^{\mathrm{a}}$ \\
\hline 101 & rs 80357220 & $\mathrm{~N}$ & CCT $[\mathrm{A} / \mathrm{T}] \mathrm{AA} \mathrm{AAG}$ & $\mathrm{K} 607^{\mathrm{a}}$ \\
\hline 102 & rs 80357239 & $\mathrm{~N}$ & TTC TG[G/A] GTG & $\mathrm{W} 1718^{\mathrm{a}}$ \\
\hline 103 & rs 80357254 & $\mathrm{~N}$ & ACA $[\mathrm{A} / \mathrm{T}] \mathrm{AA}$ TGT & $\mathrm{K} 1290^{\mathrm{a}}$ \\
\hline 104 & rs 80357269 & $\mathrm{~N}$ & TTA T[C/A $]$ A TTG & $\mathrm{S} 1262^{\mathrm{a}}$ \\
\hline 105 & rs 80357282 & $\mathrm{~N}$ & AGG $[\mathrm{A} / \mathrm{T}] \mathrm{AG}$ TCT & $\mathrm{K} 614^{\mathrm{a}}$ \\
\hline 106 & rs 80357303 & $\mathrm{~N}$ & TTG [A/T]AA GTT & $\mathrm{K} 1601^{\mathrm{a}}$ \\
\hline 107 & rs80357304 & $\mathrm{N}$ & ATA $[\mathrm{G} / \mathrm{T}] \mathrm{GA}$ GCA & $\mathrm{G} 484^{\mathrm{a}}$ \\
\hline 108 & rs 80357305 & $\mathrm{~N}$ & GAA $[\mathrm{C} / \mathrm{T}] \mathrm{AG}$ CAT & $\mathrm{Q} 1420^{\mathrm{a}}$ \\
\hline 109 & rs 80357343 & $\mathrm{~N}$ & TCC $[\mathrm{A} / \mathrm{T}] \mathrm{AA} \mathrm{CAA}$ & $\mathrm{K} 1322^{\mathrm{a}}$ \\
\hline 110 & rs 80357355 & $\mathrm{~N}$ & AAA $[\mathrm{A} / \mathrm{T}] \mathrm{AG} \mathrm{TAC}$ & $\mathrm{K} 654^{\mathrm{a}}$ \\
\hline 111 & rs 80357381 & $\mathrm{~N}$ & AAA TG[T/A] GTG & $\mathrm{C} 801^{\mathrm{a}}$ \\
\hline 112 & rs80357401 & $\mathrm{N}$ & GAA [G/T]AA TTT & E1661 ${ }^{\mathrm{a}}$ \\
\hline 113 & rs 80357424 & $\mathrm{~N}$ & AAT [G/T]AA ATA & $\mathrm{E} 1060^{\mathrm{a}}$ \\
\hline 114 & rs 80357432 & $\mathrm{~N}$ & AGA $[\mathrm{G} / \mathrm{T}] \mathrm{AA}$ TCC & $\mathrm{E} 1754^{\mathrm{a}}$ \\
\hline 115 & rs 80357440 & $\mathrm{~N}$ & TCT T[C/A]A CAG & $\mathrm{S} 1298^{\mathrm{a}}$ \\
\hline 116 & rs 80357456 & $\mathrm{~N}$ & GAG $[\mathrm{C} / \mathrm{T}] \mathrm{AA}$ AGC & Q1359 ${ }^{\mathrm{a}}$ \\
\hline 117 & rs 80357461 & $\mathrm{~N}$ & AGT [G/T]AA TTG & $\mathrm{E} 1302^{\mathrm{a}}$ \\
\hline 118 & rs 80357485 & $\mathrm{~N}$ & AAA $[\mathrm{C} / \mathrm{T}] \mathrm{AA}$ AGT & Q1096 ${ }^{\mathrm{a}}$ \\
\hline 119 & rs 138608489 & $\mathrm{~N}$ & AAA $[\mathrm{G} / \mathrm{T}] \mathrm{AA} \mathrm{CCA}$ & $\mathrm{E} 1490^{\mathrm{a}}$ \\
\hline 120 & rs 397508833 & $\mathrm{~N}$ & TGC T[C/G]A GAG & $\mathrm{S} 361^{\mathrm{a}}$ \\
\hline 121 & rs397508903 & $\mathrm{N}$ & AAA $[\mathrm{G} / \mathrm{T}] \mathrm{AA} \mathrm{TCT}$ & $\mathrm{E} 577^{\mathrm{a}}$ \\
\hline 122 & rs 80356857 & S & ACT CA[G/A] CAG & Q1395Q \\
\hline
\end{tabular}

$N$ nonsense, $M$ missense, $S$ synonymous

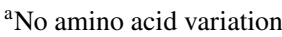


Table 2 SIFT and PROVEAN analyses prediction of 61 BRCA1 nsSNPs

\begin{tabular}{|c|c|c|c|c|c|}
\hline & Codon change & Amino acid & SIFT & PROVEAN & Mutation Taster \\
\hline 1 & ACT A[C/T]T CAT & R1699W & $\mathrm{D}$ & $\mathrm{N}$ & Del \\
\hline 2 & AAG [T/A]GT GAC & $\mathrm{C} 39 \mathrm{~S}$ & $\mathrm{D}$ & $\mathrm{N}$ & Del \\
\hline 3 & GGG C[C/G]C TTC & P1771R & $\mathrm{D}$ & $\mathrm{N}$ & Del \\
\hline 4 & CAG $[\mathrm{T} / \mathrm{G}] \mathrm{GT}$ CCT & $\mathrm{C} 61 \mathrm{G}$ & $\mathrm{D}$ & $\mathrm{N}$ & Del \\
\hline 5 & CAG [T/C]GT CCT & C61R & $\mathrm{D}$ & $\mathrm{N}$ & Del \\
\hline 6 & TTC T[G/C $] \mathrm{G} \mathrm{GTG}$ & W1718S & $\mathrm{D}$ & $\mathrm{N}$ & Del \\
\hline 7 & $\mathrm{AAC} A[\mathrm{~T} / \mathrm{G}] \mathrm{G} \mathrm{CCC}$ & M1775R & $\mathrm{D}$ & $\mathrm{N}$ & Del \\
\hline 8 & AAC A[T/A]G CCC & M1775K & $\mathrm{D}$ & $\mathrm{N}$ & Del \\
\hline 9 & GAA G[T/G]C AGA & V1736G & $\mathrm{D}$ & $\mathrm{N}$ & Del \\
\hline 10 & GAA G[T/C $] \mathrm{C}$ AGA & V1736A & $\mathrm{D}$ & $\mathrm{N}$ & Del \\
\hline 11 & CTC [G/A]AA TTA & E597K & $\mathrm{T}$ & Del & Del \\
\hline 12 & TTA T[G/A]T AAG & C64Y & $\mathrm{D}$ & $\mathrm{N}$ & Del \\
\hline 13 & CTA G[G/C]A ATT & G1706A & $\mathrm{D}$ & $\mathrm{N}$ & Del \\
\hline 14 & TCC A[C/G]A AAG & $\mathrm{T} 37 \mathrm{R}$ & $\mathrm{D}$ & $\mathrm{N}$ & Del \\
\hline 15 & ACT $[\mathrm{A} / \mathrm{G}] \mathrm{CT}$ CAT & T1686A & $\mathrm{D}$ & $\mathrm{N}$ & Del \\
\hline 16 & GAA [G/C]AG ATA & E649Q & $\mathrm{D}$ & Del & Del \\
\hline 17 & GAG TG[G/T] GTG & W1837C & $\mathrm{D}$ & $\mathrm{N}$ & Del \\
\hline 18 & AGT [G/C]AA AGA & E445Q & $\mathrm{D}$ & $\mathrm{N}$ & $\mathrm{N}$ \\
\hline 19 & CGA [G/A]AG TGG & E1836K & $\mathrm{D}$ & $\mathrm{N}$ & Del \\
\hline 20 & ACT $[\mathrm{C} / \mathrm{A}] \mathrm{AG}$ GAA & Q780K & $\mathrm{D}$ & Del & Del \\
\hline 21 & CTT [G/A]AA CTA & E $624 \mathrm{~K}$ & $\mathrm{D}$ & Del & $\mathrm{N}$ \\
\hline 22 & CTA [G/C]AG GGA & E1559Q & $\mathrm{D}$ & $\mathrm{N}$ & $\mathrm{N}$ \\
\hline 23 & GTT T[C/T]A AAG & S864L & $\mathrm{D}$ & Del & Del \\
\hline 24 & ACT [G/A]AA TTG & E638K & $\mathrm{D}$ & Del & Del \\
\hline 25 & GGA A[T/C]T GCG & $\mathrm{I} 1707 \mathrm{~T}$ & $\mathrm{D}$ & $\mathrm{N}$ & Del \\
\hline 26 & GTT A[T/G]G AAA & M1689R & $\mathrm{D}$ & $\mathrm{N}$ & Del \\
\hline 27 & GTT A[T/C $]$ G AAA & M1689T & $\mathrm{D}$ & $\mathrm{N}$ & Del \\
\hline 28 & TTA [T/G]GT AAG & $\mathrm{C} 64 \mathrm{G}$ & $\mathrm{D}$ & $\mathrm{N}$ & Del \\
\hline 29 & CTG [T/A]GT GGT & $\mathrm{C} 1787 \mathrm{~S}$ & $\mathrm{D}$ & $\mathrm{N}$ & Del \\
\hline 30 & TGT G[G/A]T GCT & G1788D & $\mathrm{D}$ & $\mathrm{N}$ & Del \\
\hline 31 & CAG T[G/A]T CCT & C61Y & $\mathrm{D}$ & $\mathrm{N}$ & Del \\
\hline 32 & CAG T[C/T]T ATT & $\mathrm{S} 1722 \mathrm{~F}$ & $\mathrm{D}$ & $\mathrm{N}$ & Del \\
\hline 33 & TGG G[T/A]G TTG & V1838E & $\mathrm{D}$ & $\mathrm{N}$ & Del \\
\hline 34 & $\mathrm{~A}[\mathrm{~T} / \mathrm{C}] \mathrm{G} \mathrm{GAT}$ & M1T & $\mathrm{D}$ & $\mathrm{N}$ & Del \\
\hline 35 & TTT T[G/T]C ATG & $\mathrm{C} 47 \mathrm{~F}$ & $\mathrm{D}$ & $\mathrm{N}$ & Del \\
\hline 36 & ACT [G/A]AG CCA & K1489E & $\mathrm{T}$ & Del & $\mathrm{N}$ \\
\hline 37 & TTG [G/A]AA GAA & E1352K & $\mathrm{D}$ & $\mathrm{N}$ & Del \\
\hline 38 & GTT $[\mathrm{A} / \mathrm{T}] \mathrm{GC}$ TAT & S1715C & $\mathrm{D}$ & $\mathrm{N}$ & Del \\
\hline 39 & TTC TG[G/T] GTG & W1718C & $\mathrm{D}$ & $\mathrm{N}$ & Del \\
\hline 40 & GCA [G/A]AA GAG & E879K & $\mathrm{D}$ & Del & Del \\
\hline 41 & GAC C[A/G]C ATA & $\mathrm{H} 41 \mathrm{R}$ & $\mathrm{D}$ & $\mathrm{N}$ & Del \\
\hline 42 & GGG C[T/C]A GAA & L1764P & $\mathrm{D}$ & $\mathrm{N}$ & Del \\
\hline
\end{tabular}


Table 2 (continued)

\begin{tabular}{llllll}
\hline & Codon change & Amino acid & SIFT & PROVEAN & Mutation Taster \\
\hline 43 & [A/G]TG GAT & M1V & D & N & ND \\
44 & TTA [G/C]AA CAG & E1419Q & D & N & ND \\
45 & TTT [T/A]GC AAA & C44S & D & N & ND \\
46 & TTT [T/G]GC ATG & C47G & D & N & Del \\
47 & AAA [A/G]GG AGC & R71G & D & N & Del \\
48 & GAA A[G/T]G TCA & R1495M & D & N & Del \\
49 & GAA A[G/A]G TCA & R1495K & D & N & Del \\
50 & TCT T[C/T]A ACC & S1596L & D & N & Del \\
51 & ATC T[T/C]A GAG & L22S & D & N & Del \\
52 & TTT T[G/T]C AAA & C44F & D & N & ND \\
53 & TTT T[G/A]C AAA & C44Y & D & N & ND \\
54 & AGA G[G/A]A GAT & G1738E & D & N & ND \\
55 & GAA A[T/G]C TGT & I1766S & D & N & ND \\
56 & AT[G/T] GAT & M1I & D & N & ND \\
57 & AAG T[G/A]T GAC & C39Y & D & N & Del \\
58 & GCT T[T/C]A ATA & L440S & D & Del & Del \\
59 & AAG T[C/T]A TTT & S988L & D & Del & N \\
60 & ATG [A/C]AA ACA & K1690Q & D & N & ND \\
61 & CTC [G/A]AA AAA & E575K & D & Del & Del \\
\hline
\end{tabular}

$B$ benign, $D$ damaging, $D e l$ deleterious, $N$ neutral, $N D$ no data, $P$ pathogenic, $T$ tolerated

Publisher's Note Springer Nature remains neutral with regard to jurisdictional claims in published maps and institutional affiliations. 